\title{
Griginal Communxations.
}

\section{REMARKS ON DR. HERBERT DAVIES'S METHOD OF TREATING ACUTE RHEUMATISM.}

By W. O. Markham, M.D., F.R.C.P., Physician to St. Mary's Hospital.

Dr. Herbert Davies recommends a new method* of treating acute rheumatic fever ; viz., " exclusively by free blistering. He has lately systematically pursued this plan in the London Hospital. The treatment is absolutely local, without the aid of "alkalies, nitre, lemon-juice, bark, opium, colchicum, or, in fact, any of the internal remedies which are and have been considered as specific in that affection." The result, Dr. Davies says, is in all respects highly satisfactory. His theory of rheumatism is the ordinarily accepted one, that there exists in the system a materies morbi, consisting either of an excess of some normal element, or of a new product, intensely acid, and highly irritating to synovial and fibrous tissues. Upon the rapid expulsion of this element depends, in his opinion, the immunity of the patient from heartdisease. Thus arguing, and believing that the virus localised itself in the affected joints, he determined to try to eliminate it there, wherever it shows local signs of its presence, bringing it out bodily with the serum of the blistered surface. "Armlets, wristlets, thighlets, leglets, and even fingerlets (if I may be allowed to coin such words) of blisters, were applied near to, but not upon, every joint inflamed, at the very height of the inflammatory stage, when the local pains were most severe, and the constitutional disturbance the gieatest.

Details of the cases (thirteen in number) on which Dr. Davies founds his opinion, are given in the London Hospital Reports. Since their publication, he has, he tells us, had further experience of the treatment, and has "obtained such valuable results as to lead him to hope that the plan suggested presents the most efficient means of rapidly subduing pain, limiting the duration, and diminishing the tendency to the production of cardiac disease."

A novel method of treating so formidable a disease as is rheumatic fever, and one so warmly recommended by an able and experienced observer like Dr. Davies, will naturally attract the attention of the profession; but I an sure no one will more readily admit than Dr. Davies that it must bear the test of experiment and much criticism before it become an accepted and established method of cure. Every man of medicine, alas! knows only too much, by sad experience, of excited hopes and fallen expectations in the matter of specific and sure cures of acute rheumatism, as well as of many other diseases. He, therefore, festinat lenté-is reasonably slow of trusting, even on such high authority, this last newly proposed remedy. Certainly the cases, thirteen in number, published by Dr. Davies as proofs of the good things he attributes to the remedy, are in my

* This treatment may fairly be called new in a relative sense; but I believe that blistering of joints suffering from acute rheumatism has been already often practised. About thirty years ago, for ex ample, it was, I think, a common practice with Dr. Duncan in the Ediuburgh Infirmary. opinion, if I may venture one, quite insufficient to prove his case. The main point, we suppose, to show of the efficacy of the remedy, is, that it prevents cardiac complications. But what satisfactory proof of such effect is given in these cases? No fewer than eight of these had signs of cardiac disease when admitted into hospital, and are therefore valueless, or nearly so, as indicators of the action of the remedy in preventing cardiac disease; so that, in reality, only five of the thirteen can be spoken of in this respect. But is it not a fact that, at the present moment, and under other (what may be called the ordinary) treatment of rheumatic fever, cardiac disease has of late become comparatively a rare occurrence? Besides, it must not be forgotten that certain individuals-young persons, for example, and especially young females-subjects of acute rheumatism, are much more liable to the cardiac complication than are grown-up men ; and, therefore, in any estimation of the value of a remedy as a preventive of heart-affection, this fact must be duly taken into calculation. But no such dllowance has here been made by Dr. Davies. Moreover, one important item -in my opinion, the most important of all-has been totally omitted in Dr. Davies's details : I mean the general management of the patient, and especially as regards keeping up the sweating by clothing him in blankets. According to my own experience, this general management of the patient is, of all remedial agencies, the most important and essential. No other remedy equally efficacious can be substituted for it. I have no hesitation in saying that I could produce just as good a list of successfully treated patients as is this of Dr. Davies, under the following simple treatment; viz., keeping the patient constantly and resolutely (for the patients often object to the discomfort of the thing) wrapped in blankets, and in a state of perspiration; giving Dover's powder for the purpose of maintaining perspiration, and in quantity sufficient to ease inordinate pain ; keeping the bowels open and the kidneys in action by small doses of saline purgatives, mixed with liquor potassæ; applying occasionally a few leeches to any joint which may be especially painful; and swathing the joints in moist flannels ; the diet being a mild milk one. I have in this way, and without the administration of alkalies or any other drugs (excepting a little coloured water, to satisfy the patient's fancy), treated the most typical cases of rheumatic fever as successfully as by any other method of treatment.

I once had great faith in the virtue of the alkaline treatment of acute rheumatism; but many broken hopes and failures in its use have long since led me to disbelieve in it as having any especial or specific efficacy. I rarely, in fact, now employ it.

It seems to me a very rational way of arguing, that, admitting there be a materies morbi in the system of the subjects of acute rheumatism, the best way to eliminate it is by assisting what seems the natural effort of the body to be rid of it; viz., the sweating. Cardiac complications were much more common in past days than they are now; and in those days the sweating was rather discouraged, as being hurtful, and as seriously weakening the body. I must, therefore, also venture to think that, touching the most important point in the treatment of this disease, Dr. Davies says nothing-i.e., he lays no stress upon it: I mean upon the encouragement of the perspiration.

The proposition, that the materies morbi is especially concentrated in substance in the inflamed joints, is, of course, at present an hypothesis-a reasonable, and, it may be, a true hypothesis. But, before proof, it is certainly just as rational a one, that the morbific element is distributed throughout the whole body- 
i.e., wherever the blood circulates; and, therefore, on the principle of treatment adopted in this case by Dr. Davies-viz., by the process of its eliminationit is assuredly as rational to encourage the elimination per vias naturales, that is, by the sudoriparous glands, as it is through the serum of the blistered surfaces of the inflamed joints. The serum of even many blistered joints must in amount be very greatly less than the perspiration morbidly excited by the disease, and actively encouraged by artificial means, from the whole cutaneous surface of a rheumatic patient.

The application of blisters to the joints, as recommended by Dr. Davies, may be certainly considered a good and rational proposition in the treatment of acute rheumatism, and will, no doubt, on his recommendation, be fully tried. But it should surely, at present, at all events, be only regarded as an adjunct to other and far more important treatment. To regard it, as Dr. Davies seems to do, as the primum mobile in the cure-as a sort of specific methodus curandi of acute rheumatism-is what I have here ventured to object to in my remarks upon Dr. Davies's papers.

\section{ON CHOREA, AND ON NATURE AND ART IN THE TREATMENT.}

By Jalies Turnbulu, M.D., Physician to the Liverpool Royal Infirmary.

[Read before the Liverponl Medical Society, December 1st, 1964.]

Chorea, or St. Vitus's Dance, is well known as a nervous affection of spasmodic nature, manifesting itself by irregular contractions or twitchings of the voluntary muscles, which the patient cannot control. It occurs much more frequently in females than in males; and more commonly between the ages of 8 and 15 than in younger children or grown-up persons. Those attacked are not usually of strong constitution, but of nervous temperament and sensitive disposition; and fright is often the exciting cause. $I$ have observed that in females it occurs most fre quently just before the catamenial function is established; and, when it comes on after this, there is generally some menstrual irregularity, accompanied with more or less anæmia. I have also observed the occurrence of worms in connection with the disease; but intestinal irritation has not often appeared to me to be the sole cause.

The connection between chorea and rheumatism is one of the most singular facts in relation to this disease, and I have had many opportunities of observing it in private, as well as in hospital practice. We frequently see chorea coming on while the joints are swelled and painful from an attack of acute or subacute rheumatism, and it appears in such cases to supersede the rheumatic affection. Again, we see cases of chorea where the joints become swelled and painful from rheumatism, supervening or recurring. In either case, the one disease seems to take the place of the other. Besides this, we find that chorea occurs frequently in families where there is the rheumatic diathesis, one member being attacked with chorea, and others liable to rheumatic attacks. Chorea is a functional disorder, and in fatal cases no organic alterations which could be the cause of the disease have been discovered, but the organic effects which rheumatic fever prodaces on the heart and its valves have been not unfrequently observed. As we have good reason to believe that rheumatism is produced by a materies morbi in the blood, the close connection between rheumatism and chorea suggests the inquiry whether the latter might not be dependent on the same or a similar cause. Some countenance is also given to such a theory, by the fact that when urea is retained in the blood in consequence of disease of the kidneys, there is a liability to convulsive and other nervous affections, and also by the fact that some poisons, such as strychnia, when introduced into the blood, excite spasm of the muscles.

With respect to the relation in which chorea stands to other diseases of the nervous system, I would observe that it bears to paralysis this relation, that we sometimes find in chorea not only that the patient is unable to use the muscles of the tongue so as to speak, but that it is impossible to use some of the limbs, owing to a kind of temporary imperfect paralysis. It bear's also to paralysis this further relation, that in some cases one side only of the body is affected. Epilepsy is a more intractable and severe disease, at tended with protracted muscular spasm and complete loss of consciousness, and ultimate injury of the mental powers, which never occur in chorea; but the absence of any traceable organic alteration of the brain or cord characterises both. As regards severity, chorea holds an intermediate place between epilepsy and hysteria, the convulsive form of which latter is more troublesome than dangerous. I have seen some cases, however, where chorea and hysteria approximated so closely, that they might not inappropriately be called cases of hysterical chorea.

Chorea is often a very trifling complaint, but it is also frequently a troublesome protracted disease, and in a few instances I have seen it prove fatal. It is then one of the most frightful diseases we can witness, causing violent contortions, and tossing the patient about in all directions, thus injuring and abrading all the prominent parts of the body, and causing rapid loss of flesh and death from exhaustion, owing to incessant motion and want of sleep. Whenever the patient sleeps, the twitchings cease, and thus the strength is renewed; but if the movements should be so violent as to preclude sleep, the prognosis becomes very grave.

The disease often hegins with slight twitching, and gradually attains its highest degree of severity; but very severe cases may recover more rapidly than slighter ones. I had two cases under my care at the same time this summer which illustrate this; one was a girl of 12 years of age, who was brought regulirly to see me at my house. Though the case was never very severe, it was much longer in recovering than the other, which was the most severe in which I have seen recovery take place. As to the duration of the disease, Dr. Hughes, in his digest of one hundred cases, tells us that, exclusive of the time the patients had been previously ill, it varied in Guy's Hospital from three weeks to three months, 40 per cent. of the cases being cured in from three to six weeks.

The general diffusion amongst medical men of the present day of a knowledge of the important fact that in all curable diseases there is a spontaneous tendency to recovery, is a distinguishing feature of our times ; and, when combined with a due appreciation of the influence of art in promoting this object, such knowledge must exercise a most beneficial influence on practice. Sir John Forbes's little treatise is the only one in which we have a comprehensive examination of this subject, and whilst I wouid observe that I place a high value on the service which he rendered by his exposition of the power of nature in the cure of disease, I must add that I think the tendency of his work is to repress, and unduly repress, the progress of medicine in the direction of therapeutic inquiry. 